\title{
Reduction of lattice thermal conductivity from planar faults in the layered Zintl compound $\mathrm{SrZnSb}_{2}$
}

\author{
$\varnothing$. Prytz, ${ }^{1, a)}$ E. Flage-Larsen, ${ }^{1}$ E. S. Toberer, ${ }^{2}$ G. J. Snyder, ${ }^{2}$ and J. Taft ${ }^{1}$ \\ ${ }^{1}$ Department of Physics, University of Oslo. P. O. Box 1048 - Blindern, NO-0316 Oslo, Norway \\ ${ }^{2}$ California Institute of Technology, Materials Science, 1200 East California Boulevard, Pasadena, California \\ 91125, USA
}

(Received 10 October 2010; accepted 24 December 2010; published online 24 February 2011)

\begin{abstract}
The layered Zintl compound $\mathrm{SrZnSb}_{2}$ is investigated using transmission electron microscopy (TEM) to understand the low lattice thermal conductivity. The material displays out-of-phase boundaries with a spacing from 100 down to $2 \mathrm{~nm}$. Density functional theory calculations confirm that the TEM-derived defect structure is energetically reasonable. The impact of these defects on phonon scattering is analyzed within the Debye-Callaway model, which reveals a significant reduction in the acoustic phonon mean free path. This enhancement in phonon scattering leads to an $\sim 30 \%$ reduction in lattice thermal conductivity at $300 \mathrm{~K}$. C 2011 American Institute of Physics. [doi:10.1063/1.3549821]
\end{abstract}

\section{INTRODUCTION}

Thermoelectric materials require the unusual combination of a high Seebeck coefficient $(\alpha)$ coupled with low electrical resistivity $(\rho)$ and low thermal conductivity $(\kappa)$. Together, these properties are expressed in the material figure of merit ${ }^{1}, z t=\alpha^{2} T /(\rho \kappa)$. Zintl compounds have recently attracted significant attention as thermoelectric materials due to their low lattice thermal conductivity and valence precise nature. ${ }^{2}$ Good thermoelectric performance has been realized in a number of Zintl compounds, including $\mathrm{Yb}_{14}, \mathrm{MnSb}_{11}$, $\mathrm{CeCoFe}_{3} \mathrm{Sb}_{12}, \mathrm{SrZn}_{2} \mathrm{Sb}_{2}$, and $\mathrm{CsBi}_{4} \mathrm{Te}_{6}{ }^{3-6}$

The latter two compounds are particularly intriguing; these are layered compounds that show high charge carrier mobility. Phonon transport in layered compounds can similarly be attractive for thermoelectric materials. At the extreme, layered materials with turbostratic disorder have shown some of the lowest thermal conductivity values measured in a solid. ${ }^{7}$

In our pursuit of new Zintl compounds for thermoelectric applications, we have previously investigated $\mathrm{SrZnSb}_{2}{ }^{8}$ This compound crystallizes in the orthorhombic space group Pnma, with unit cell axes $a=23.05 \AA$, $b=4.37 \AA$, and $c=4.46 \AA{ }^{9}$ ' The structure is formed by layers of $\mathrm{Sr}, \mathrm{Sb}$, and Zn stacked along the $a$ axis [Fig. 1(a)] with adjacent layers of $\mathrm{Sb}$ and $\mathrm{Zn}$ atoms forming tetrahedra, see Fig. 2 of Ref. 9. The structure and bonding of layered Zintl compounds are extensively discussed by Burdett and Miller. ${ }^{10}$ Our prior investigation of $\mathrm{SrZnSb}_{2}$ found the lattice thermal conductivity to be unusually $\operatorname{low}^{8}\left(1 \mathrm{~W} \mathrm{~m}^{-1} \mathrm{~K}^{-1}\right.$ at $\left.400 \mathrm{~K}\right)$. This value is about half of what has been observed in, e.g., the filled Skutterudite compounds. ${ }^{11}$ While this low lattice thermal conductivity can largely be attributed to the complex unit cell (26 atoms in the primitive cell), we also speculated whether microstructural features such as twinning may be present and contribute to the low lattice thermal conductivity.

\footnotetext{
a) Author to whom correspondence should be addressed. Electronic mail: oystein.prytz@fys.uio.no.
}

In light of the small difference in the $b$ and $c$ lattice parameters, twinning is certainly conceivable, and planar faults are known to influence the lattice thermal conductivity through phonon scattering. ${ }^{12}$

In the present study we perform transmission electron microscopy (TEM) investigations of the $\mathrm{SrZnSb}_{2}$ compound. We show that planar defects are present, but that these are not due to twinning. Instead, the defects are out-of-phase boundaries created by rigid shifts in the $b-c$ plane of the material. These defects are discussed in light of the similar $\mathrm{SrZnBi}_{2}$ compound, and we report on calculations of the energetics of the observed defects. Furthermore, the effect of

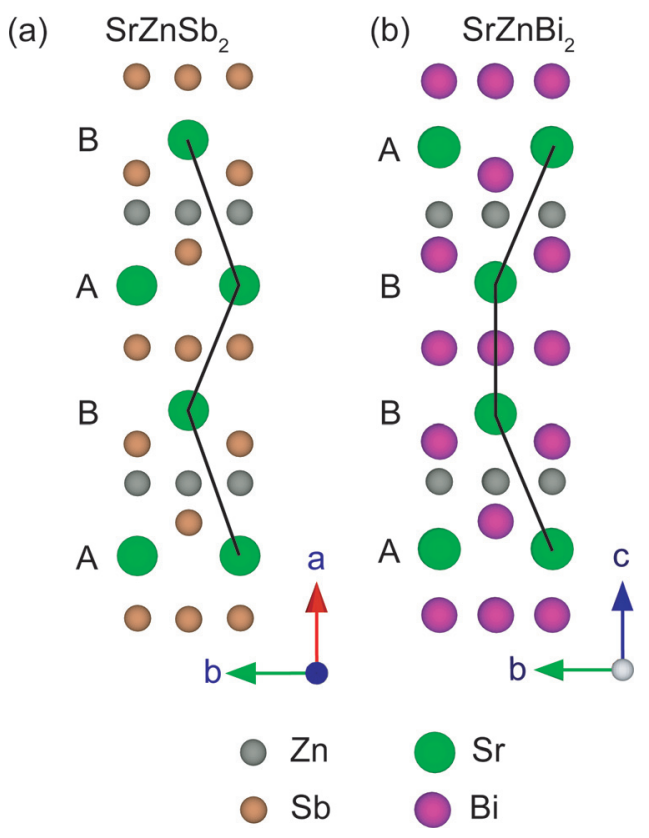

FIG. 1. (Color online) The crystal structure of (a) $\mathrm{SrZnSb}_{2}$ and (b) $\mathrm{SrZnBi}_{2}$. The arrangement of $\mathrm{Zn}$ and $\mathrm{Sb} / \mathrm{Bi}$ atoms is very similar in the two structures, while the sequence of $\mathrm{Sr}$ atoms is different. A rigid shift of half the unit cell by the vector $\left[\begin{array}{lll}0 & 1 / 2 & 1 / 2\end{array}\right]$ would give similar arrangement of $\mathrm{Sr}$ atoms, while leaving the $\mathrm{Zn}$ and $\mathrm{Sb} / \mathrm{Bi}$ arrangement virtually unchanged. 


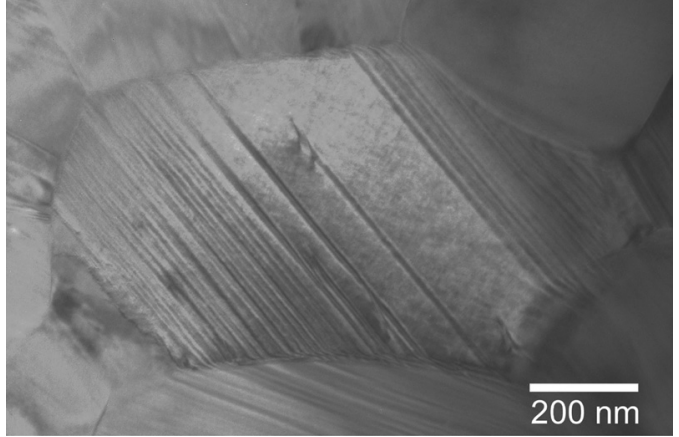

FIG. 2. Bright field TEM micrograph showing the observed planar defects.

the observed defects on the lattice thermal conductivity is assessed using a Debye-Callaway model.

\section{EXPERIMENTAL AND THEORETICAL TECHNIQUES}

$\mathrm{SrZnSb}_{2}$ was prepared from stoichiometric amounts of the elements (at least $99.9 \%$ metal basis purity) following the method described in Ref. 8. The elements were combined in a pyrolitic $\mathrm{BN}$ crucible, which was sealed in a quartz tube evacuated to about $10^{-3} \mathrm{~Pa}$, and kept at $1225 \mathrm{~K}$ for $30 \mathrm{~min}$. The resulting ingot was ball milled under argon for $15 \mathrm{~min}$ in the high energy SPEX 8000 Series Mixer/Mill utilizing stainless steel vial and balls. The fine grained, homogeneous powder obtained from milling was hot pressed in a high-density graphite die (POCO) at $825 \mathrm{~K}$, for approximately $3 \mathrm{~h}$, while roughly 1.4 metric tonnes of force was placed on a 12-mm-diam surface. Specimens for TEM were prepared by standard mechanical grinding and ion milling techniques. The specimens were studied in JEOL 2000FX and 2010F transmission electron microscopes, both operated at $200 \mathrm{kV}$.

The energetics of the observed defects were calculated within the framework of density functional theory as implemented in the Vienna Ab-initio Simulation Package (VASP). ${ }^{13,14}$ The calculations employed the Perdew-BurkeErnzerhof ${ }^{15}$ exchange-correlation functional in the generalized gradient approximation. An energy cutoff of $550 \mathrm{eV}$ and a $\Gamma$-centered $k$-point sampling of $3 \times 15 \times 15$ were sufficient to converge the total energies. Models of the observed planar faults were generated, and the structure was relaxed in cell shape, volume, and atomic positions using a residual minimization, direct inversion in the iterative subspace scheme. ${ }^{16}$ The resulting total energy was compared with the total energies of pristine material based on both the experimental and relaxed $\mathrm{SrZnSb}_{2}$ cell parameters and atomic positions.

\section{RESULTS AND DISCUSSION}

\section{A. The defect structure of $\mathrm{SrZnSb}_{2}$}

The TEM studies revealed a universally present faulted structure. Virtually all observed grains displayed planar defects similar to those of Fig. 2. The distance between these defects varied somewhat. The typical distance was somewhat below $100 \mathrm{~nm}$ in width, while the smallest observed spacing was only a couple of nanometers, which is close to the unit cell size. In projections where the electron beam was perpendicular to the $a$ axis, the contrast from the defects disappeared, only to reappear when the sample was slightly tilted so that the electron beam was inclined relative to the $a$ axis. Figure 3 shows an example of this where we note the absence of the defects contrast in the $01 \overline{1}$ projection (a), while the contrast reappears when the sample is tilted about the [011] axis (b).

These observations indicate that the planar defects lie in the $b-c$ plane of the crystal, and that the observed contrast is mainly due to a phase shift of the electrons in regions where the crystal on either side overlap.

Selected area electron diffraction tilt series revealed no additional or split reflections, features that would be expected in the case of twin domains. However, we observed systematic streaks in the diffraction patterns, see figure 4. This streaking is parallel to the $\mathbf{a}^{*}$ axis and appears for reflections with indices $k+1=2 n+1$. Such features are typical for planar defects where the structure is shifted a vector $\mathbf{r}$ across the boundary. In the presence of such defects, streaking will appear ${ }^{17}$ if the phase shift $(\varphi)$ across the boundary differs from an integer number of $2 \pi$, that is

$$
\varphi=2 \pi \mathbf{g} \cdot \mathbf{r} \neq 2 \pi n
$$

Thus, we see that the observations are consistent with a shift vector $\mathbf{r}=\left[\begin{array}{lll}0 & 1 / 2 & 1 / 2\end{array}\right]$, while shifts of, e.g., [ [ $\left.\begin{array}{lll}0 & 1 / 2 & 0\end{array}\right]$, $\left[\begin{array}{lll}0 & 0 & 1 / 2\end{array}\right]$, or $\left[\begin{array}{lll}0 & 1 / 4 & 1 / 4\end{array}\right]$ would not explain the observations.

Comparing the structure of orthorhombic $\mathrm{SrZnSb}_{2}$ [Fig. 1(a)] with the tetragonal $\mathrm{SrZnBi}_{2}$ compound [Fig. 1(b)],
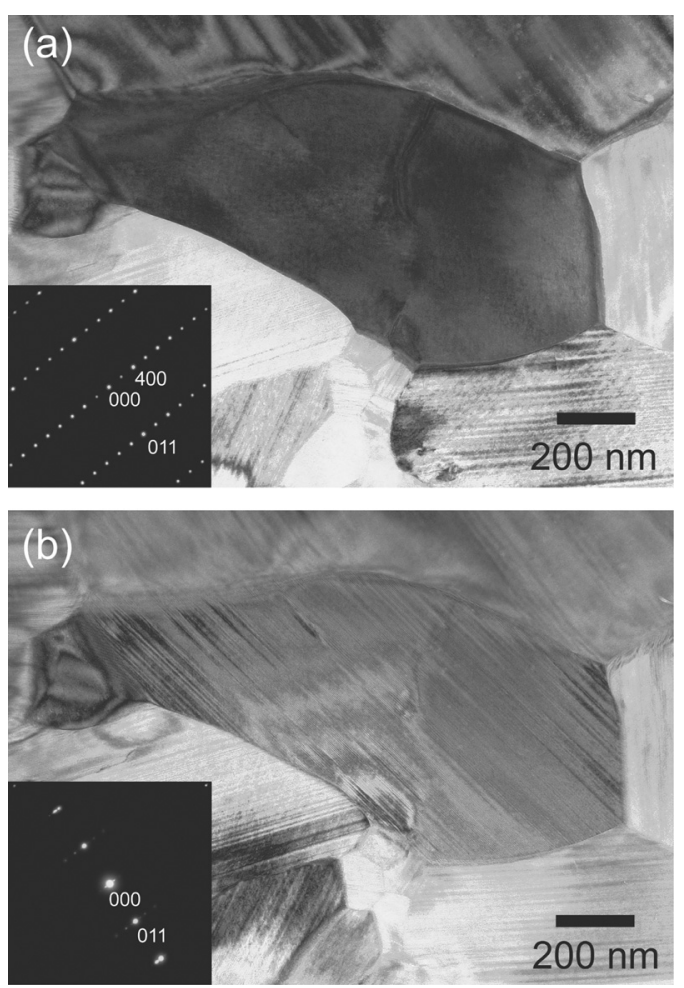

FIG. 3. (a) Bright field image of a grain in the $00 \overline{1}$ projection. Note the absence of domain contrast. (b) The same grain tilted about the $\left[\begin{array}{lll}0 & 1 & 1\end{array}\right]^{*}$ axis so that the electron beam was inclined to the $\mathbf{a}^{*}$ axis. In this orientation the domain's overlap and contrast were observed. 
we see that there are striking similarities. This is not surprising as $\mathrm{Sb}$ and $\mathrm{Bi}$ are chemically very similar elements. While the $\mathrm{Zn}$ and $\mathrm{Sb} / \mathrm{Bi}$ atoms occupy very similar positions in the two structures, there are some obvious differences with regard to the $\mathrm{Sr}$ atoms. In the $\mathrm{SrZnSb}_{2}$ system, the $\mathrm{Sr}$ atoms form a zig-zag sequence along the long $a$ axis, with each layer shifted $\left[\begin{array}{lll}0 & 1 / 2 & 1 / 2\end{array}\right]$ relative to the previous. The sequence of $\mathrm{Sr}$ atoms can be considered as a stacking of the form $\mathrm{ABAB}$. In comparison, the $\mathrm{Sr}$ atoms in the $\mathrm{Bi}$ system are stacked head to head before a shift occurs, giving a stacking of the form ABBA. Thus the main structural difference between the materials is the configuration of the three layers lying in the middle of the unit cell. In Fig. 5, an idealized model of the $\mathrm{SrZnSb}_{2}$ system is shown, focusing on these three layers. We see that a shift $\mathbf{r}=\left[\begin{array}{lll}0 & 1 / 2 & 1 / 2\end{array}\right]$ in the Sr layer will align the $\mathrm{Sr}$ atoms. Thus the stacking of $\mathrm{Sr}$ atoms in the faulted material will be of the form ABBA, thereby changing the structure locally to resemble the Bi-containing compound, albeit with an orthorhombic distortion.

Calculations using density functional theory confirmed that these defects are associated with a very small increase in energy. Models with and without the defects were generated consisting of $2 \times 1 \times 1$ unit cells. For the defect model, the atoms in the range $x=[0.51,1.49]$ were shifted by the observed shift vector, giving a two-defect model. In Table I we have listed the total energy of these models, calculated both with and without a structural relaxation. From the calculations that include a structural relaxation, we see that the observed defects are associated with an energy of $20.6 \mathrm{~mJ} / \mathrm{m}^{2}$. In comparison, the frequently appearing (111) antiphase boundary in $\mathrm{Ni}_{3} \mathrm{Al}$ has an energy more than eight times larger, ${ }^{18}$ at $180 \mathrm{~mJ} / \mathrm{m}^{2}$. It should therefore be expected that planar defects of the type proposed here could easily arise in the $\mathrm{SrZnSb}_{2}$ system, for example due to thermal or mechanical stresses.

\section{B. Impact on the lattice thermal conductivity}

Prior investigation of the lattice thermal conductivity and speed of sound of $\mathrm{SrZnSb}_{2}$ found extremely low $\kappa_{L}$ of $1.2 \mathrm{~W} \mathrm{~m}^{-1} \mathrm{~K}^{-1}$ and $v_{m}=2270 \mathrm{~m} / \mathrm{s}$ at room temperature. ${ }^{8}$ In contrast, the chemically similar compound $\mathrm{SrZn}_{2} \mathrm{Sb}_{2}$ exhibited almost twice the lattice thermal conductivity, despite
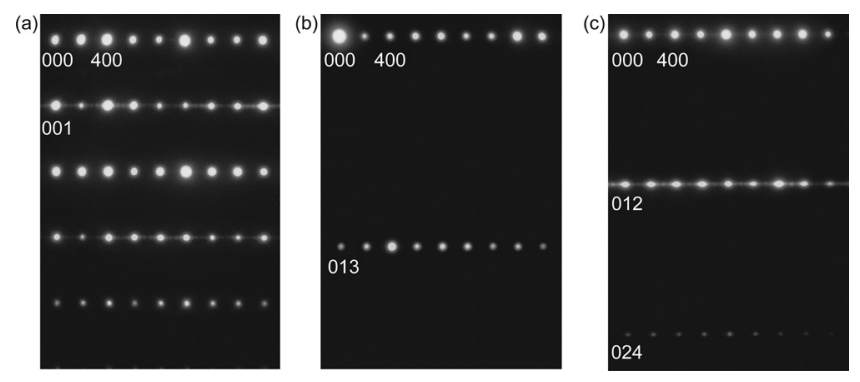

FIG. 4. Selected area electron diffraction patterns obtained by tilting about the $\mathbf{a}^{*}$ axis. (a) The 010 projection, (b) the $03 \overline{1}$ projection, (c) the $02 \overline{1}$ projection. The streaks appear for reflections $k+1=2 n+1$, indicating a shift $\mathbf{r}=\left[\begin{array}{lll}0 & 1 / 2 & 1 / 2\end{array}\right]$. The sample thickness causes multiple scattering, resulting in some kinematically forbidden reflections appearing, e.g., the 001 reflection. The inset in (c) shows a possible ordering of the domains.
TABLE I. Calculations of the total energy for models with and without the observed defect. The calculations were performed both with the experimental lattice parameters and atomic positions as input, and for the relaxed structure. A $2 \times 1 \times 1$ unit cell model was used, and for the defect calculations the middle part was shifted by the experimentally observed shift vector $\left[\begin{array}{lll}0 & 1 / 2 & 1 / 2\end{array}\right]$, giving two defects in the model.

\begin{tabular}{lll}
\hline \hline Model & Unrelaxed & Relaxed \\
\hline Defect free model & $-107.62 \mathrm{eV}$ & $-107.71 \mathrm{eV}$ \\
Two defect model & $-107.53 \mathrm{eV}$ & $-107.66 \mathrm{eV}$ \\
Energy per defect & $0.0450 \mathrm{eV}$ & $0.0250 \mathrm{eV}$ \\
& $37.0 \mathrm{~mJ} / \mathrm{m}^{2}$ & $20.6 \mathrm{~mJ} / \mathrm{m}^{2}$ \\
\hline
\end{tabular}

having a similar acoustic speed of sound. This prior work attributed this difference to the difference in unit cell complexity (5 and 16 atoms in the primitive unit cells of $\mathrm{SrZn}_{2} \mathrm{Sb}_{2}$ and $\mathrm{SrZnSb}_{2}$, respectively). The optical modes are assumed to carry minimal heat due to their frequent scattering and low group velocity. Note that the difference in mean free path reported in Ref. 8 partially arises from the difference in high frequency cut-off for each acoustic branch.

With the TEM observations described here, it is intriguing to revisit the prior investigation of the lattice thermal conductivity of $\mathrm{SrZnSb}_{2}$ and $\mathrm{SrZn}_{2} \mathrm{Sb}_{2}$, which are shown in Fig. 5(a). A simple Callaway model for the lattice thermal conductivity can be employed [Eq. (2)], consisting of frequency dependent terms for the heat capacity $C(\omega)$ and the phonon relaxation time $\tau(\omega)$ and a group velocity $v$ set by the speed of sound:

$$
\kappa_{L}=\frac{1}{3} v^{2} \int_{0}^{\omega_{a}} C(\omega) \tau(\omega) d \omega .
$$

For this Debye solid, the maximum frequency considered will be the maximum acoustic frequency $\omega_{a}$, as the optical modes are considered only a minor source of heat conduction. The effective phonon relaxation time will arise from Umklapp $\left(\tau_{U}\right)$ and boundary $\left(\tau_{B}\right)$ scattering, as shown in the following:

$$
\tau^{-1}=\tau_{U}^{-1}+\tau_{B}^{-1} .
$$

In a polycrystalline material, the boundaries can be approximated as the average grain diameter $d$, leading to a $\tau_{B}=d / v$. Developing an expression for $\tau_{U}$ is slightly more involved. As summarized in Ref. 19, $\tau_{U}$ may be

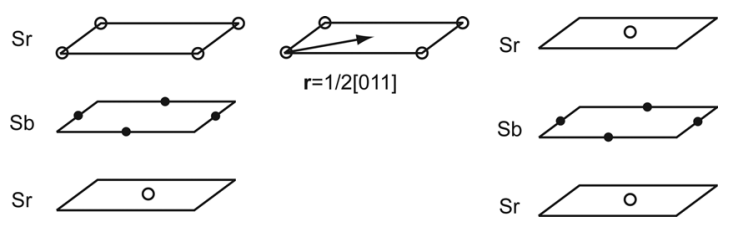

FIG. 5. Sketch of the middle three layers of the $\mathrm{SrZnSb}_{2}$ structure (left). The suggested shift (middle) places the top Sr layer above the bottom $\mathrm{Sr}$ atoms, giving a structure which locally resembles that of the $\mathrm{SrZnBi}_{2}$ structure (right). 
approximated using Eq. (4) with average atomic mass $M$, Debye temperature $\theta_{D}$, and Gruneisen parameter $\gamma$ :

$$
\tau_{U}^{-1} \approx \frac{\hbar \gamma^{2}}{M v^{2} \theta_{D}} \omega^{2} T \exp \left(-\theta_{D} / 3 T\right) .
$$

We note that this expression typically invokes a prefactor of order of unity and should be similar for related materials. The prefactor in Eq. (4) is evaluated using the Callaway model and the known experimental lattice thermal conductivity at $300 \mathrm{~K}$ of $\mathrm{SrZn}_{2} \mathrm{Sb}_{2}$. The boundary scattering in this polycrystalline material is on the order of the grain size ( $1 \mu \mathrm{m})$, as no nanoscale features were observed by TEM.

Assuming the $\tau_{U}$ prefactor is similar between $\mathrm{SrZn}_{2} \mathrm{Sb}_{2}$ and $\mathrm{SrZnSb}_{2}$, the frequency dependent $\tau_{U}$ for $\mathrm{SrZnSb}_{2}$ is calculated and shown in Fig. 6(b). If polycrystalline $\mathrm{SrZnSb}_{2}$ did not possess any additional disorder at the nanoscale, a $d$ of $1 \mu \mathrm{m}$ would be appropriate. Such parameters would overestimate $\kappa_{L}$ of $\mathrm{SrZnSb}_{2}$, yielding $1.5 \mathrm{~W} / \mathrm{mK}$ at $300 \mathrm{~K}$. Indeed, the work here suggests that $d$ is less than $0.1 \mu \mathrm{m}$ due to the structural disorder inherent in $\mathrm{SrZnSb}_{2}$. The effective $\tau$ curves [Eq. (3)] are shown in Fig. 6(b) for these two scenarios. The model which includes the observed nanostructuring $(d=0.1 \mu \mathrm{m})$ predicts a value of $1.1 \mathrm{~W} / \mathrm{mK}$, in agreement with the experimental data. Within the accuracy of these models, the nanostructures observed here yield an $\sim 30 \%$ reduction in $\kappa_{L}$. The difference in thermal conductivity between $\mathrm{SrZnSb}_{2}$ and $\mathrm{SrZn}_{2} \mathrm{Sb}_{2}$ thus arises from structural
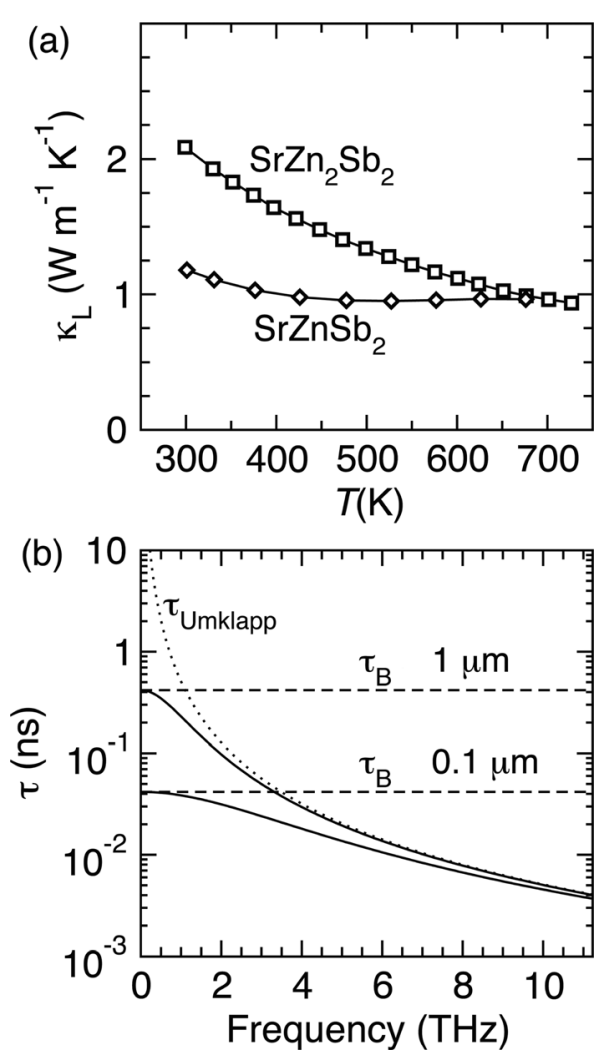

FIG. 6. (a) Lattice thermal conductivity of $\mathrm{SrZnSb}_{2}$ is significantly lower than $\mathrm{SrZn}_{2} \mathrm{Sb}_{2}$ near room temperature, reported in Ref. 8. (b) Phonon relaxation time $(\tau)$ for $\mathrm{SrZnSb}_{2}$ calculated from Eq. (3) with contribution from boundary $\left(\tau_{B}\right)$ and Umklapp scattering [Eq. (4)] at $300 \mathrm{~K}$. Nanoscale boundaries associated with stacking faults significantly reduce $\tau$. complexity which increases the amount of heat trapped in optical modes and from nanostructures which reduce the phonon relaxation time.

The use of an energy-independent boundary scattering model is clearly a major simplification, as long wavelength phonons (those with wavelengths beyond the boundary length) are unlikely to be scattered by these out-of-phase boundaries. The effect of this scattering is quite small, however, as the lattice thermal conductivity contribution from phonons with wavelength in excess of $100 \mathrm{~nm}$ is $\sim 1 \%$ of the total lattice thermal conductivity calculated using an Umklapp scattering-dominated Debye-Callaway model.

\section{SUMMARY AND FINAL DISCUSSIONS}

In summary, the $\mathrm{SrZnSb}_{2}$ compound displays outof-phase boundaries corresponding to rigid shifts of the unit cell in the $b-c$ plane (shift vector $\mathbf{r}=\left[\begin{array}{lll}0 & 1 / 2 & 1 / 2\end{array}\right]$ ). These defects are associated with a small increase in the total energy of the system. Furthermore, the defects cause an increased phonon scattering, which results in a reduction in the acoustic phonon mean free path, thereby contributing significantly to the low lattice thermal conductivity of this material.

Scattering from out-of-phase boundaries is a rather intriguing method to develop thermoelectric materials. Charge carriers passing through these boundaries encounter coherent interfaces, presumably leading to minimal reduction in mobility. Phonons encountering these interfaces likewise do not encounter roughness or elastic contrast but do experience a breakdown of periodicity, leading to scattering and a reduction in lattice thermal conductivity. Layered materials that can accommodate such coherent extended defects may thus be a "gentle" way to engineer high mobility, low lattice thermal conductivity thermoelectric materials. Complex materials, which offer more stacking options, should show a greater propensity to form such defects compared to relatively simple materials.

\section{ACKNOWLEDGMENTS}

The authors would like to thank A. F. May of Caltech for synthesis and discussions, and A. Olsen of the University of Oslo for discussions.

${ }^{1}$ G. J. Snyder and E. S. Toberer, Nature Mater. 7, 105 (2008).

${ }^{2}$ E. S. Toberer, A. F. May, and G. J. Snyder, Chem. Mater. 22, 624 (2010). ${ }^{3}$ E. S. Toberer, C. A. Cox, S. R. Brown, T. Ikeda, A. F. May, S. M. Kauzlarich, and G. J. Snyder, Adv. Funct. Mater. 18, 2795 (2008).

${ }^{4}$ B. C. Sales, D. Mandrus, and R. K. Williams, Science 272, 1325 (1996).

${ }^{5}$ E. S. Toberer, A. F. May, B. C. Melot, E. Flage-Larsen, and G. J. Snyder, Dalton Trans. 39, 1046 (2010).

${ }^{6}$ D. Y. Chung, T. Hogan, P. Brazis, M. Rocci-Lane, C. Kannewurf, M. Bastea, C. Uher, and M. G. Kanatzidis, Science 287, 1024 (2000).

${ }^{7}$ C. Chiritescu, D. G. Cahill, N. Nguyen, D. Johnson, A. Bodapati, P. Keblinski, and P. Zschack, Science 315, 351 (2007).

${ }^{8}$ A. F. May, E. S. Toberer, and G. J. Snyder, J. Appl. Phys. 106, 013706 (2009).

${ }^{9}$ E. Brechtel, G. Cordier, and H. Schäfer, Z. Naturforsch. B 34B, 251 (1979).

${ }^{10}$ J. K. Burdett and G. J. Miller, Chem. Mater. 2 , 12 (1990).

${ }^{11}$ G. S. Nolas, J. L. Cohn, and G. A. Slack, Phys. Rev. B 58, 164 (1998). 
${ }^{12}$ D. L. Medlin and G. J. Snyder, Curr. Opin.Colloid Interface Sci. 14, 226 (2009).

${ }^{13}$ G. Kresse and J. Hafner, Phys. Rev. B 47, 558 (1993).

${ }^{14}$ G. Kresse and J. Furthmüller, Phys. Rev. B 54, 11169 (1996).

${ }^{15}$ G. Kresse and D. Joubert, Phys. Rev. B 59, 1758 (1999).

${ }^{16}$ P. Pulay, Chem. Phys. Lett. 73, 393 (1980).
${ }^{17}$ G. Thomas and M. J. Goringe, Transmission Electron Microscopy of Materials (Wiley, New York, 1979), Chap. 8.

${ }^{18}$ A. J. Skinner, J. V. Lill, and J. Q. Broughton, Modell. Simul. Mater. Sci. Eng., 3, 359 (1995).

${ }^{19}$ J. Yang, in Thermal Conductivity: Theory, Properties, and Applications, edited by T. M. Tritt (Plenum, New York, 2004), Chap. 1.1. 